\title{
Effect of Vitamin Restriction on Cecal Microflora and Fermentation in Mice
}

\author{
Yoshiyuki MoRISHITA \\ Department of Biomedical Food Research, National Institute of Health, Toyama 1-23-1, \\ Shinjuku-ku, Tokyo 162
}

(Received for publication, July 21, 1994)

\begin{abstract}
Experiments were conducted to examine the effect of different vitamin mixture contents in a diet on the cecal microflora and short-chain fatty acid concentrations in malc ICR mice. Mice fed a vitamin-free dict showed a profound increase in the numbers of enterobacteriaceae and enterococci and a significant increase in bacteroidaceae in the cecum, while they showed no remarkable changes in the numbers of lactobacilli, bifidobacteria and anaerobic gram-positive cocci. Short-chain fatty acid (SCFA) concentrations were profoundly reduced by the vitamin-free regimen. Mice on a $0.3 \%$ vitamin diet showed significant decreases in enterobacteriaceae and enterococci and a significant increase in bifidobacteria as compared to animals on a $1 \%$ vitamin dict (basal) although their body wcight gain was approximately $60 \%$ of the animals fed the basal or $2 \%$ vitamin diet. SCFAs showed decreased concentrations; in particular butyrate and valerate were significantly reduced in the $0.3 \%$ vitamin group compared to the basal group. The $2 \%$ vitamin mixture group showcd no significant differences from the $1 \%$ vitamin group in cecal microflora composition and SCFA concentrations.
\end{abstract}

Key words: vitamin; cecal microflora; fermentation; mice; restriction

Food restriction has been shown to retard the aging processes of experimental animals, extend their life span $(13,15,17)$ and reduce the incidence of spontaneous or induced cancer $(16,17)$. In addition, the nutritional conditions as well as aging have been reported to influence the gastrointestinal microflora $(1,2,10,14)$. Thus, the previous studies dealt with the effect of food restriction and vitamin- or calorierestriction on the cecal microflora of rats $(8,9)$. Consequently, it was shown that food restriction and vitamin deficiency resulted in increased numbers of bifidobacteria and lactobacilli and reduced production of short-chain fatty acids (SCFAs) with no remarkable changes in aerobic populations in the cecum of all the rats except one. This could be ascribed to the fecal refection by coprophagy in the rat. As shown by a previous report (12), there are differences between the mouse and rat in the response of microflora to dietary substances. Therefore, the vitamin restriction would have different effects on the intestinal microflora between mice and rats. In addition, undernourished children have been reported to have abnormal intestinal microflora $(3,4)$, which might be associated with vitamin deficiency. The purpose of the present investigation 


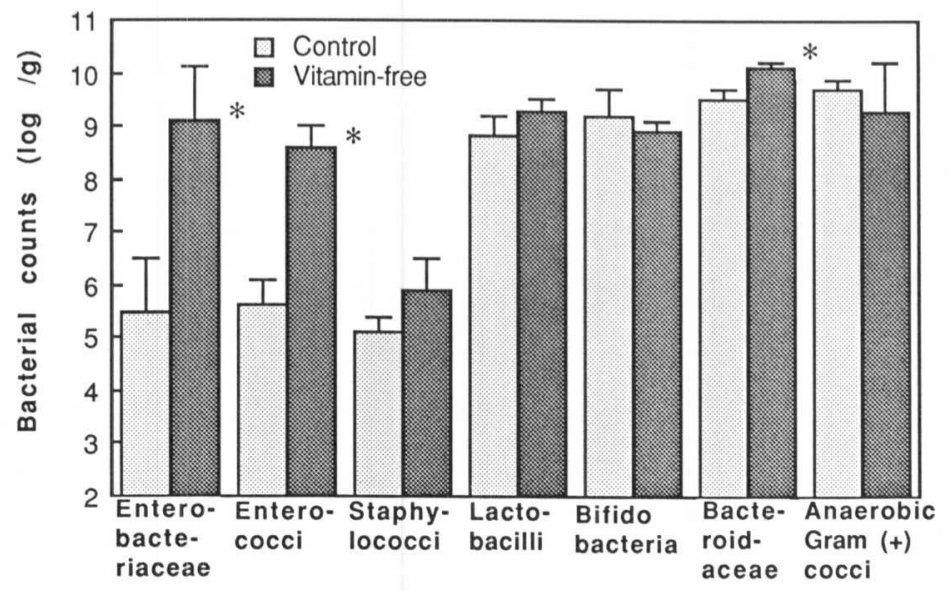

Fig. 1. Cecal microflora of mice fed vitamin-free diet.

*Significant difference from the $1 \%$ vitamin group $(p<0.05)$.

was to demonstrate the effect of different vitamin contents on the cecal microflora and SCFA concentrations in mice.

\section{MATERIALS AND METHODS}

Animals and diets. Animals used were 4-week-old male ICR mice from Shizuoka Laboratory Animal Center (Shizuoka, Japan). Five mice were allocated to each cage, which was metallic and with stainless steel mesh bottom, and allowed free access to the basal or experimental diets and water after 4-days acclimation on the basal diet. Ten mice were used for each experimental group and the body weights of them recorded every week.

Purified diets were prepared as previously described $(11,12)$. In experiment 1 animals were fed a purified diet with or without $1 \%$ vitamin mixture; in experiment 2 animals were fed a purified diet with $0.3,1$, or $2 \%$ vitamin mixture.

Sampling and bacteriological techniques. Animals were killed with dry ice after 3 or 4 weeks of feeding. The cecal contents were aseptically collected. Half of the animals were used for bacteriological analysis and the other half to measure $\mathrm{pH}$ and SCFA concentrations. Preparation of media and dilution of samples were made as described in previous reports $(5,10)$. Anaerobic cultivation was performed at $37^{\circ} \mathrm{C}$ in steel jars with a piece of steel wool covered with reduced copper in an atmosphere of $10 \% \mathrm{CO}_{2}$ and $90 \% \mathrm{~N}_{2}$. Colony counting was performed and bacteria were broadly classified as previously described (10).

pH and SCFA measurements. $\mathrm{pH}$ value and SCFA concentrations were determined according to the procedure of Morishita et al. $(7,12)$.

Statistics. All data are expressed as means with standard deviations and were analyzed by Student's $t$-test. 
Table 1. Effect of vitamin deficiency on cccal SCFA concentrations in mice

\begin{tabular}{ccc}
\hline & \multicolumn{2}{c}{ Vitamin content } \\
\cline { 2 - 3 } & $0 \%$ & $1 \%$ (basal) \\
\hline Acids $(\mu$ mol/g) & & \\
Total acids & $24.2 \pm 2.2^{*}$ & $42.5 \pm 3.6$ \\
Acetic & $14.4 \pm 1.2^{*}$ & $23.4 \pm 1.9$ \\
Propionic & $4.4 \pm 0.5^{*}$ & $8.5 \pm 1.0$ \\
n-Butyric & $2.3 \pm 0.4^{*}$ & $6.6 \pm 0.8$ \\
n-Valerate & $0.7 \pm 0.2^{*}$ & $1.1 \pm 0.2$ \\
iso-Butyric & $0.7 \pm 0.2^{*}$ & $1.4 \pm 0.5$ \\
iso-Valeric & $1.6 \pm 0.4$ & $1.5 \pm 0.6$ \\
Cecal pH & $8.0 \pm 0.1^{*}$ & $7.7 \pm 0.1$ \\
Body weight gain $(\mathrm{g})^{\mathrm{b}}$ & $1.1 \pm 0.4^{*}$ & $27.9 \pm 1.9$ \\
\hline
\end{tabular}

Data are mean $\pm \mathrm{SD}$ ( ${ }^{\mathrm{a}} n=5$ for acid concentrations and $\mathrm{pH} ;{ }^{\mathrm{b}} n=10$ for body weight gain).

*Significant difference from the $1 \%$ group $(p<0.05)$.

\section{RESULTS}

\section{Effect of Vitamin-Free Diet on Cecal Microflora and SCFA Concentrations (Experiment 1)}

As seen in Fig. 1, enterobacteriaceae and enterococci showed an overgrowth (9.1 and 8.6, respectively, in average $\log _{10}$ count) in the cecum of mice fed a vitamin-free diet and staphylococci increased in number to some extent, but not significantly, when compared with animals fed a control (basal) diet. Bacteroidaceae (10.1) also significantly increased in number and lactobacilli showed a tendency to increase in the vitamin-free diet. Anaerobic gram (+) cocci (AGPG) and bifidobacteria did not show any significant change in number in the vitamin-free diet.

SCFA concentrations, except iso-valerate, were remarkably lowered by the vitamin-free regimen (Table 1). Especially butyrate showed the most remarkable decrease in concentration $(2.3 \mu \mathrm{mol} / \mathrm{g})$ in the vitamin-free group compared to the control group $(5.5 \mu \mathrm{mol} / \mathrm{g})$. Mice on the vitamin-free diet manifested malignant malnutrition with no body weight gain for 4 weeks.

Effect of Various Concentrations of Vitamin Mixture in Diet on Cecal Microflora and SCFA Concentrations (Experiment 2)

Figure 2 shows the cecal microflora of ICR mice fed diets with different contents of vitamin mixture in experiment 2. Enterobacteriaceae were found in the smallest numbers ( 4.7 in average $\log _{10}$ counts) in the $0.3 \%$ vitamin group compared with the other groups and showed a significantly decreased number in the $2 \%$ vitamin compared with the $1 \%$ vitamin group. Enterococci showed a tendency to vary in proportion to the vitamin content. In contrast, the numbers of bifidobacteria and bacteroidaceae tend to be inversely proportional to the vitamin content of diets. Particularly bifidobacteria showed a significant increase in the $0.3 \%$ vitamin group as compared to the $1 \%$ vitamin group. Lactobacilli showed a tendency to decrease in number in the $2 \%$ 


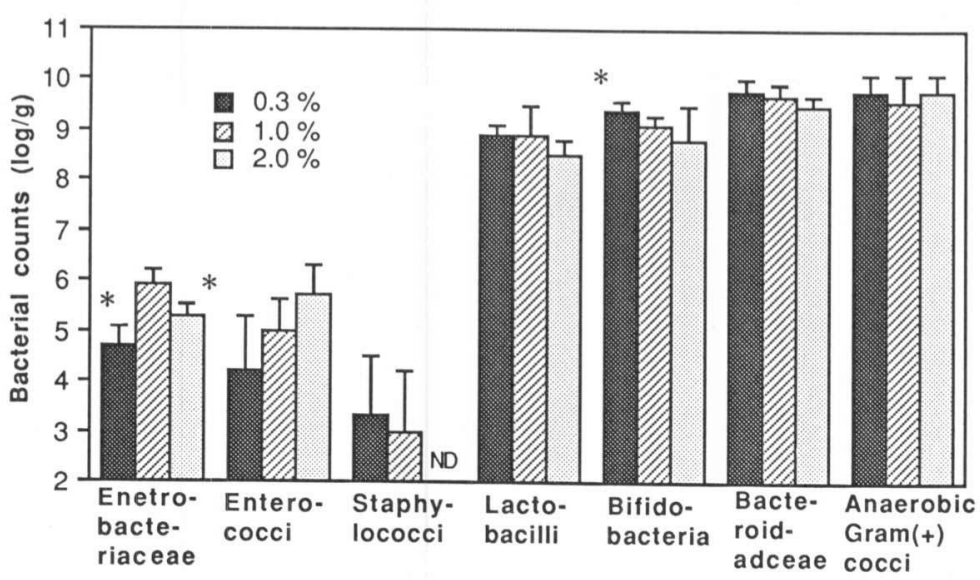

Fig. 2. Cecal microflora of mice fed $0.3 \%, 1 \%$, or $2 \%$ vitamin-containing diet.

*Significant difference from the $1 \%$ vitamin group $(p<0.05)$. ND, not detected.

Table 2. Effect of vitamin contents on cecal SCFA concentrations in mice

\begin{tabular}{|c|c|c|c|}
\hline & \multicolumn{3}{|c|}{ Vitamin content } \\
\hline & $0.3 \%$ & $1 \%$ (basal) & $2 \%$ \\
\hline \multicolumn{4}{|l|}{ Acids $(\mu \mathrm{mol} / \mathrm{g})^{\lambda}$} \\
\hline Total acids & $41.5 \pm 6.9$ & $49.1 \pm 9.2$ & $45.4 \pm 6.3$ \\
\hline Acetic & $24.6 \pm 4.0$ & $27.1 \pm 5.3$ & $25.3 \pm 4.3$ \\
\hline Propionic & $8.5 \pm 1.7$ & $10.3 \pm 3.2$ & $9.3 \pm 1.5$ \\
\hline n-Butyric & $5.3 \pm 1.1^{*}$ & $7.8 \pm 1.3$ & $6.6 \pm 0.9$ \\
\hline n-Valerate & $0.9 \pm 0.2^{*}$ & $1.3 \pm 0.2$ & $1.2 \pm 0.1$ \\
\hline iso-Butyric & $1.2 \pm 0.2$ & $1.6 \pm 0.4$ & $1.7 \pm 0.3$ \\
\hline iso-Valeric & $1.2 \pm 0.2$ & $1.1 \pm 0.2$ & $1.4 \pm 0.3$ \\
\hline Cecal $\mathrm{pH}^{\mathrm{a}}$ & $7.9 \pm 0.2$ & $7.9 \pm 0.1$ & $8.0 \pm 0.1$ \\
\hline Body weight gain $(g)^{b}$ & $12.8 \pm 1.4$ & $22.4 \pm 1.9$ & $20.3 \pm 0.8$ \\
\hline
\end{tabular}

Data are mean $\pm \mathrm{SD}\left({ }^{\mathrm{a}} n=5\right.$ for acid concentrations and $\mathrm{pH} ;{ }^{\mathrm{b}} n=10$ for body weight gain).

*Significant difference from the $1 \%$ group $(p<0.05)$.

vitamin group.

As shown in Table 2, SCFA concentrations reduced in the $0.3 \%$ vitamin group compared to the others although there were no significant differences in the total acid concentration between the 3 experimental groups. Butyrate and valerate, however, were significantly reduced in the $0.3 \%$ group compared to the $1 \%$ group. The valerate concentration also was significantly different from the $2 \%$ group. The rate of the $0.3 \%$ group to the $1 \%$ group was $68 \%$ for butyrate and $69 \%$ for the valerate concentration, which were much lower than the rates for acetic and propionic acids. The $\mathrm{pH}$ values showed no differences between the 3 groups. Body weight gain for 20 days was $12.3 \mathrm{~g}(57 \%$ weight of the $1 \%$ vitamin diet), $22.4 \mathrm{~g}$ and $20.3 \mathrm{~g}$ for mice on the $0.3 \%$, $1 \%$ and $2 \%$ vitamin diets, respectively. 


\section{DISCUSSION}

The mice fed a vitamin-free diet showed the overgrowth of aerobic bacteria such as enterobacteriaceae and enterococci, that is, the abnormal microflora in the cecum (experiment 1). Mice were more susceptible to a vitamin-free regimen than rats previously reported (9). This might be ascribed to no or little fecal refection due to poor corprophagy behavior in mice as manifested by no body weight gain in mice on the vitamin-free diet. Escherichia coli and enterococci are suppressed by chloroform-resistant bacteria (CRB) as reported by Morishita and Mitsuoka (6). Vitamin-free regimen, therefore, may be considered to result in the diminished population and/or activity of $\mathrm{CRB}$ in mice, which is also suggested by the fact that vitamin deficiency resulted in the most remarkable reduction of butyrate in the cecum of mice. This is similar to the phenomenon observed in the rat (9). It is important that bifidobacteria as well as lactobacilli maintained the same population level even in the vitamin-free group as that in the control (experiment 1). These bacteria probably utilized vitamins from the metabolisms of other intestinal bacteria. Mata et al. (4) found that facultatively anaerobic bacteria were increased in the small intestine with a lower frequency of bifidobacteria in children with severe protein-calorie malnutrition. The present study suggests that besides protein-calorie malnutrition, vitamin deficiency and other factors derived from malnutrition might be associated with the abnormal intestinal microflora in undernourished children.

Bacteroidaceae were found in significantly increased numbers in the vitamin-free group compared to the $1 \%$ vitamin group in experiment 1 , and in experiment 2 the numbers of them were in somewhat inverse proportion to the vitamin content, which suggest that the vitamin content influences the growth of bacteroidaceae in the cecum of mice. This tendency in mice was not found in rats previously reported (9). In addition, the present data indicate that the vitamin shortage may have a suppressive effect on anaerobic bacteria producing butyrate and valerate (experiment 2).

Interestingly, the mice on the $0.3 \%$ vitamin diet had smaller numbers of enterobacteriaceae and enterococci and larger numbers of bifidobacteria in their cecum than the other groups (experiment 2). Similar observations were previously obtained from rats fed the identical diets with different contents of vitamin mixture (9). This increase of bifidobacteria might be related to the decreased activity of bacteria that produce butyrate and assimilate mannitol since rats on dietary mannitol showed a decreased number of bifidobacteria and an increased concentration of cecal butyrate (7). Actually, among the SCFAs, butyrate showed the largest reduction in concentration, which was about $70 \%$ of the cecal butyrate concentration in $1 \%$ vitamin-fed mice. It is likely that $0.3 \%$ vitamin mixture in a diet may be sufficient for mice to maintain the normal cecal microflora although their weight gain for 20 days was $57 \%$ and $63 \%$ of that of animals fed $1 \%$ and $2 \%$ vitamin, respectively. The present and previous data suggest that a $1 \%$ vitamin mixture in a purified diet is necessary for the maximum growth of mice and rats but not necessary for maintenance of the cecal microflora.

The suppressed growth of enterobacteriaceae and enterococci may be beneficial to 
the host since these bacteria are potential pathogens. The suppression of these bacteria may be associated with the enhanced growth or activities of fermentative bacteria such as bifidobacteria and lactobacilli. Additionally, the $0.3 \%$ vitamin regimen might be not inhibitive to the maintenance of CRB although SCFA concentrations reduced to some extent in the cecal contents of mice. Actually the total SCFA concentration in the cecal contents of mice on the $0.3 \%$ vitamin regimen was approximately $85 \%$ of that in $1 \%$ vitamin-fed mice. The present data showed that the vitamin-free regimen induced abnormal microflora in mice, which differed from the microflora in rats on the identical vitamin-deficient diet and that of mice on a $0.3 \%$ vitamin diet had a cecal microflora similar to that in rats $(9)$.

\section{REFERENCES}

(1) Benno, Y., and T. Mitsuoka. 1991. Effect of diet and aging on human fecal microflora. Bifidobacteria Microflora 10: 89-96.

(2) Donaldson, R.M. 1964. Normal bacterial populations of the intestine and their relation to intestinal function. New Engl. J. Med. 270: 938-945.

(3) Gorbach, S.L. 1971. Intestinal microflora. Gastroenterology 60: 1110-1129.

(4) Mata, L.J., M.L. Mejicsanos, and F. Jimenez. 1972. Studies on the indigenous gastro-intestinal flora of Guatemalan children. Am. J. Clin. Nutr. 25: 1380-1390.

(5) Mitsuoka, T., T. Sega, and S. Yamamoto. 1965. Eine verbesserte Methodik der qualitativen und quantitativen Analyse der Darmflora von Menschen und Tieren. Zbl. Bakteriol. Parasit. Infektionkr. Hyg. Abt. I. Orig. A195: 455-469.

(6) Morishita, Y., and T. Mitsuoka. 1976. Microorganisms responsible for controlling the populations of Escherichia coli and enterococcus and the consistency of cecal contents in the chicken. Jpn. J. Microbiol. 20: 197-202.

(7) Morishita, Y. 1994. The effect of dietary mannitol on the cecal microflora and short-chain fatty acids in rats. Lett. Appl. Microbiol. 18: 27-29.

(8) Morishita, Y. 1995. Effect of food restriction on the cecal microflora and short-chain fatty acid concentrations in rats. Microb. Ecol. Health Dis. 8: 35-39.

(9) Morishita, Y. 1995. Effect of vitamin- and calorie-restriction on the caecal microflora and shortchain fatty acid concentrations in rats. Microb. Ecol. Health Dis. 8: 9-14.

(10) Morishita, Y., and K. Miyaki. 1979. Effects of age and starvation on the gastrointestinal microflora and the heat resistance of fecal bacteria in rats. Microbiol. Immunol. 23: 455-470.

(11) Morishita, Y., and K. Shiromizu. 1987. Effects of dietary lactose and purified diet on intestinal microflora of rats. Jpn. J. Med. Sci. Biol. 40: 15-26.

(12) Morishita,Y., H. Yamada, K. Shiiba, N. Kimura, and H. Taniguchi. 1993. Effect of hydrolyzate of wheat bran hemicellulose on the cecal microflora and short-chain fatty acid concentrations in rats and mice. Bifidobacteria Microflora 12: 19-24.

(13) Mosoro, E.J. 1988. Food restriction in rodents: an evaluation of its role in the study of aging. J. Gerontol. 43: B59-B64.

(14) Smith, H.W. 1965. Observations on the flora of the alimentary tract of animals and factors afftecting its composition. J. Pathol. Bacteriol. 89: 95-122.

(15) Snyder, D.L., M. Pollard, B.S. Wostmann, and P. Luckert. 1990. Life span, morphology, and pathology of diet-restricted germ-free and conventional Lobund-Wistar rats. J. Gerontol. 45: B52B58.

(10) Steinbach, G., S.P. Kumar, B.S. Reddy, M. Lipkin, and P.R. Holt. 1993. Effects of caloric restriction and dietary fat on epithelial cell proliferation in rat colon. Cancer Res. 3: 2745-2749.

(17) Weinduch, R. 1989. Dietary restriction, tumors, and aging in rodents. J. Gerontol. 44: 67-71. 